\title{
Develando Terra Incognita. Una búsqueda arqueológica de las primeras ocupaciones humanas en los salares de Infieles y Pedernales (3000-4100 msnm, $25^{\circ}-26^{\circ}$ S), Región de Atacama, Chile
}

Patricio López Mendoza, Carlos Carrasco González, Rodrigo Loyola Muñoz, Valentina Flores-Aqueveque, Francisca Santana-Sagredo, Antonio Maldonado Castro e Ismael Martínez Rivera

Recibido 24 de julio 2020. Aceptado 13 de noviembre 2020

\begin{abstract}
RESUMEN
Se presentan los resultados de prospecciones superficiales realizadas en los salares de Infieles $\left(25^{\circ} \mathrm{S}-69^{\circ} \mathrm{O}\right)$ y Pedernales $\left(26^{\circ} \mathrm{S}-69^{\circ} \mathrm{O}\right)$ en la Región de Atacama (Chile), en cotas de altitud que van desde los ca. 3000 a 4100 msnm. Ambas prospecciones, en zonas que conectan directamente al Norte Árido con el Semiárido, forman parte de un programa de evaluación de áreas con potenciales ocupaciones humanas durante la transición Pleistoceno-Holoceno y fueron dirigidas a sectores específicos como terrazas fluviales, abanicos aluviales, humedales y playas de salares. La variabilidad del registro arqueológico se discute en el marco de la secuencia prehispánica e histórica regional, y en particular, en el contexto de las primeras ocupaciones humanas en ambos salares.
\end{abstract}

Palabras clave: Pleistoceno-Holoceno; Prospección; Salares australes; Región de Atacama.

\section{Unveiling Terra Incognita. An archaeological search for the first human occupations of Infieles and Pedernales salt flats (3000-4100 masl, $\left.25^{\circ}-26^{\circ} \mathrm{S}\right)$, Atacama Region, Chile}

\begin{abstract}
The results of archaeological surface surveys carried out on Infieles $\left(25^{\circ} 58^{\prime} 39^{\prime} \mathrm{S}-69^{\circ} 03^{\prime} 25^{\prime \prime} \mathrm{W}\right)$ and Pedernales $\left(26^{\circ} 13^{\prime} 59^{\prime \prime} \mathrm{S}-69^{\circ} 07^{\prime} 16^{\prime \prime} \mathrm{W}\right)$ salt flats, Atacama, Chile, located at an altitude from 3000 to 4100 masl are presented. Both surveys were carried out in areas that directly connect the Arid North with the Semiarid. The surveys are part of a project that investigates areas with potential human occupations during the Pleistocene-Holocene transition and are targeted at specific sectors such as river terraces, alluvial fans, wetlands, and salt flats. The variability of the archaeological record is discussed within the framework of the regional pre-Hispanic and historic sequence, and especially within the context of the first human occupations of the salt flats.
\end{abstract}

Keywords: Pleistocene-Holocene; Archaeological survey; Southern salt flats; Atacama Region.

\footnotetext{
Patricio López Mendoza. Museo de Historia Natural y Cultural del Desierto de Atacama. Avenida O'Higgins s/n sector Parque El Loa, Calama, Chile. E-mail: patriciolopezmend@gmail.com

Carlos Carrasco González. Arqueólogo Independiente. Colegio de Arqueólogos. E-mail: carrcag@gmail.com

Rodrigo Loyola Muñoz. Instituto de Arqueología y Antropología, San Pedro de Atacama, Universidad Católica del Norte, Gustavo Le Paige 380, San Pedro de Atacama, Chile: E-mail: rodarkeo@gmail.com

Valentina Flores-Aqueveque. Departamento de Geología, Facultad de Ciencias Físicas y Matemáticas, Universidad de Chile, Plaza Ercilla 803, Santiago, Chile. E-mail: vfloresa@uchile.cl

Francisca Santana-Sagredo. Escuela de Antropología, Pontificia Universidad Católica de Chile. E-mail: francisca.santana@uc.cl Antonio Maldonado Castro. Centro de Estudios Avanzados en Zonas Áridas. E-mail: antonio.maldonado@ceaza.cl Ismael Martínez Rivera. Centro de Estudios del Hombre Austral, Instituto de la Patagonia, UMAG. E-mail: ismart68@gmail.com
}

Intersecciones en Antropología 22(1), enero-junio: 11-23. 2021. ISSN-e 1850-373X

https://doi.org/10.37176/iea.22.1.2021.558

Facultad de Ciencias Sociales - UNICEN - Argentina 


\section{INTRODUCCIÓN}

Los estudios sobre los procesos de colonización de paisajes deshabitados consideran que los ambientes de altura conllevan un alto riesgo, ya que estos representan verdaderas barreras biogeográficas para la dispersión humana, sobre todo en periodos tempranos de la prehistoria (Gamble, 1993). Para el caso de los Andes, esta idea ha influenciado al estudio del poblamiento inicial, y postula que dicho proceso se desarrolló más tardíamente durante el Holoceno temprano (Yacobaccio, 2017). No obstante, el registro en la última década de contextos arqueológicos con fechas del Pleistoceno tardío y emplazados en cotas superiores a los $3800 \mathrm{msnm}$ ha reabierto la discusión, dando paso a un panorama mucho más diverso de lo supuesto (AlbarracinJordan y Capriles, 2011; Capriles y AlbarracinJordan, 2013; Rademaker et al., 2014; Capriles et al., 2016).

En el Norte Árido de Chile $\left(18^{\circ}-27^{\circ} \mathrm{S}\right)$, en la ecorregión de la puna (3000-4100 msnm), predominan condiciones de extrema aridez, dada la contigüidad con el desierto de Atacama, por lo que no es de extrañar que tempranamente la costa se concibiese como el principal foco de asentamiento (Llagostera et al., 2000). En la actualidad se reconoce que en la puna las condiciones ambientales fueron más favorables posteriormente al Último Máximo Glacial (UMG). Diversos estudios indican dos eventos de cambio ambiental conocidos como Central Andean Pluvial Event I y II (CAPE I y II), los cuales desencadenaron un incremento de los regímenes de lluvias de verano y la recarga de acuíferos en el flanco oeste andino (Latorre et al., 2013). Como resultado, en las cuencas precordilleranas y altiplano del desierto de Atacama (2000-4000 msnm) se formaron extensos lagos, paleohumedales y vegas que concentraron una alta diversidad de recursos disponibles durante un lapso temporal discreto (12.700-9700 años cal AP; CAPE II) (Quade et al., 2008; Latorre et al., 2013).

La puna meridional del desierto de Atacama (Región de Atacama, $25^{\circ}-28^{\circ} \mathrm{S}$ ) representa un área clave para reconstruir las rutas de dispersión y los procesos de colonización en ambientes de altura, ya que es un área muy poco estudiada. Debido a este vacío de información y en función de evaluar su conexión con áreas vecinas y dinámicas ocupacionales durante la transición Pleistoceno-Holoceno, se elaboró un programa de investigación arqueológica para los salares de Pedernales, Infieles, Maricunga,
Laguna Negro Francisco y la cuenca alta del río Jorquera. Este programa busca responder la siguiente pregunta: ¿qué rol cumplieron los paisajes de altura propios de la puna meridional del desierto de Atacama durante la etapa de colonización humana inicial y también a lo largo de todas las ocupaciones prehispánicas? Para esto, el presente trabajo aborda los resultados obtenidos en las dos primeras fases de dicho programa de investigación: en primera instancia, los datos recopilados de las prospecciones arqueológicas superficiales de los salares de Infieles y Pedernales; y en segundo, los resultados de datos cronológicos de paisajes de potencial interés para ocupaciones de la transición Pleistoceno-Holoceno como bordes de lagunas y terrazas fluviales de Pedernales. Ambas cuencas -que presentan dimensiones y accesibilidad disímiles, aunque con ambientes similares- son enclaves relevantes para entender el poblamiento inicial de las tierras altas por su carácter transicional entre las manifestaciones culturales del Norte Árido y Semiárido, así como la costa del Pacífico y de la vertiente atlántica de los Andes.

\section{ANTECEDENTES}

\section{Marco arqueológico general}

Como señalamos, los ambientes de altura de los Andes brindaron una serie de recursos que podrían haber favorecido la dispersión humana temprana a lo largo del desierto de Atacama. Un escenario plausible es que las tierras altas constituyeran espacios internodales de tránsito entre ambas vertientes; o bien que representaran áreas de concentración de recursos que fueron explotadas estacionalmente dentro de sistemas de asentamientos más amplios a lo largo del ciclo anual y que incluyeron zonas situadas a menor altitud. Una tercera posibilidad es que las tierras altas fueran ocupadas permanentemente a lo largo del ciclo anual por grupos humanos con respuestas culturales efectivas para este tipo de ambientes (Osorio, 2013).

Los datos disponibles permiten sostener que durante la transición Pleistoceno-Holoceno se desarrolló el ya mencionado CAPE II, evento que incrementó la recarga de aguas subterráneas dando lugar a extensos paleohumedales en fondos de cuencas y vegas del piso prepuna (2600 a 3200 msnm) (Grosjean, Núñez y Cartajena, 2005; Quade et al., 2008). Sin embargo, al sur de los $25^{\circ}$, las condiciones ambientales cambian y la zonificación vertical 
de pisos ecológicos dependientes de las Iluvias de verano bajo los 4000 msnm es reemplazada por el denominado desierto absoluto y vegetación xerófita, relacionada con las Iluvias invernales (Maldonado et al., 2005). Hasta aproximadamente los 9500 años cal AP existió una mayor influencia del sistema de precipitaciones de los alisios desde la vertiente amazónica y que incrementó las Iluvias (Kull, Grosjean y Veit, 2002), por lo que sería esperable un aumento de la biomasa sobre los $3000 \mathrm{msnm}$.

En las tierras altas, los paleohumedales, lagos $y$ vegas constituyeron microambientes productivos pero dispersos en un contexto de aridez pronunciada, por lo que fueron hábitats apropiados para la dispersión humana (Núñez, Grosjean y Cartajena, 2002). Si se considera la hipótesis del corredor norte-sur a lo largo de las tierras altas, cabría esperar una ocupación temprana de estas áreas que sostuviera el flujo poblacional hacia el Semiárido. Por ende, es probable, para nuestra área de estudio, que los grupos humanos seleccionaran ambientes similares durante el Pleistoceno tardíoHoloceno temprano, con campamentos residenciales emplazados cercanos a los recursos críticos. En ambientes de baja productividad, la estructura de recursos en parches separados por intervalos áridos habría incentivado una alta movilidad residencial (Kelly, 2003), y una subsecuente rápida dispersión humana en los paisajes altoandinos. Considerando las restricciones ambientales, se ha planteado que estos espacios fueron ocupados por medio de una movilidad logística para la ejecución de tareas especializadas durante la época estival, en el caso de la puna seca (Osorio et al., 2011). En el caso de la puna salada, se han sugerido circuitos de movilidad residencial entre las tierras bajas, la prepuna y puna (Núñez et al., 2002), y como parte de mecanismos de fusión-fisión de bandas (Aldenderfer, 1998). De acuerdo con modelos etnográficos, los grupos de cazadores-recolectores en ambientes de baja productividad tienden a mantener una baja demografía, amplios circuitos de movilidad y una distribución dispersa en el espacio (Kelly, 1995). Sin embargo, esto implicaría un incremento de los home-range, lo que limita la posibilidad de establecer redes de interacción a larga distancia (Binford, 2001), aspecto que evaluaremos en las discusiones.

\section{Área de estudio: características generales}

El área de estudio comprendió dos grandes cuencas: Infieles y Pedernales (Figura 1a). El salar de Infieles $\left(25^{\circ} \mathrm{S}\right)$ se localiza a una altitud promedio de 3520 msnm y presenta un tamaño de cuenca de $293 \mathrm{~km}^{2}$, mientras que Pedernales $\left(26^{\circ} \mathrm{S}\right)$ se ubica a unos 3356 msnm y está emplazado en el centro de una cuenca de $3620 \mathrm{~km}^{2}$. En el sector abundan vicuñas, guanacos, zorros, roedores y aves acuáticas. La vegetación es propia de la región de la alta estepa y el clima es del tipo desierto frío de montaña, con una gran sequedad del aire y temperaturas reguladas por la altitud. Actualmente, las precipitaciones son bajas (150-200 mm anuales), con temperaturas que oscilan enormemente durante el día y que en la noche alcanzan hasta $-30^{\circ} \mathrm{C}$.

Los únicos datos arqueológicos para Infieles corresponden a un reporte de Latcham (1938), quien describe pinturas rupestres detalladas posteriormente por Cervellino (1982). Para el caso de Pedernales, los datos arqueológicos provienen de Estudios de Impacto Ambiental, en los que se reportaron diversos hallazgos prehispánicos e históricos (Castelleti, 2011; Olivares et al., 2013). Por su parte, González y Castells (2007, como se citó en Algoritmos, 2011) reportaron 22 evidencias arqueológicas en Pedernales, que van desde eventos de talla hasta un camino de posible origen incaico. En momentos históricos, ambas cuencas fueron y siguen siendo explotadas para la extracción de recursos minerales, como atestigua la mina de bórax en Pedernales, utilizada desde la segunda mitad del siglo XIX, y diversas estancias que siguen siendo utilizadas por la vigente comunidad colla en Pedernales.

\section{METODOLOGÍA DE PROSPECCIÓN Y REGISTRO}

La prospección fue realizada mediante un muestreo aleatorio estratificado para el cual las áreas fueron seleccionadas según las probabilidades de hallazgo. Esta fase de prospección corresponde a una estrategia de muestreo que contempla pasos secuenciales de investigación. En primera instancia, la identificación superficial de sitios arqueológicos; el estudio cronológico y geoarqueológico del avance y retroceso de las cuencas de salares y fluviales de ambas áreas; el muestreo de materias primas en zonas de abanicos aluviales, depresiones, secuencias estratigráficas expuestas, etc.; la elaboración de una línea base isotópica en plantas y animales; por último, el sondeo y datación de los sitios arqueológicos afines a nuestros objetivos. El presente artículo aborda los resultados de las prospecciones 
superficiales y los datos preliminares del estudio geaorqueológico de Pedernales para entender la dinámica de formación de paisajes de la transición Pleistoceno-Holoceno.

En función del análisis de información geomorfológica y ambiental se definieron áreas potenciales con mayor probabilidad de hallazgos. Estas corresponden a playas de salar de pendiente baja; abanicos aluviales con formación de paleosuelos; humedales y vegas asociados al borde de los salares; aleros y oquedades en ignimbrita observados en distintos sectores de ambos salares, terrazas y desembocaduras de quebradas, sectores de llanos, y terrazas fluviales del río Juncalito en Pedernales hasta la cota de $4100 \mathrm{msnm}$. Complementariamente, se sondearon una serie de paleohumedales y terrazas fluviales con material orgánico datable mediante ${ }^{14} \mathrm{C}$, sobre todo perfiles expuestos para identificar paleosuelos y zonas de humedales actualmente desaparecidos.

El área prospectada fue de $16 \mathrm{~km}^{2}$ para el caso de Infieles y de $18 \mathrm{~km}^{2}$ en Pedernales. Los distintos sectores fueron cuadriculados a partir de cuadrantes de $1 \mathrm{~km}^{2}$, en los que a su vez se distribuyeron transectas paralelas, separadas cada $50 \mathrm{~m}$. Los hallazgos fueron geoposicionados mediante el Datum WGS84 y clasificados según periodificaciones generales: Prehispánico, Prehispánico-Histórico, Histórico e Indeterminado. Se diferenció entre sitios arqueológicos y hallazgos aislados, estos últimos definidos a partir de tres o menos evidencias arqueológicas superficiales.

De acuerdo con las geoformas seleccionadas, cada salar fue dividido en grandes sectores. Esto favoreció una mayor diversidad de paisajes y de unidades de muestreo abordados para el caso de Pedernales, cuenca que posee una dimensión 12 veces superior a Infieles. En el caso de este último salar, los sectores corresponden a: Sector A, playa ubicada al norte del salar y al este de la ignimbrita; Sector B, ignimbritas y playa al norte del salar; Sector C, abanicos aluviales, desembocaduras de quebrada, junto con playas al sur del salar y al oeste de la ignimbrita; Sector $\mathbf{D}$, abanico aluvial y playas de salar localizados al oeste; y Sector E, playas ubicadas al sur del salar (Figura 1b).

En Pedernales se definieron los siguientes sectores: Sector A, terrazas lacustres al noreste del salar; Sector B, cuenca de la quebrada de Pedernales y playas del salar ubicadas al suroeste; Sector C,
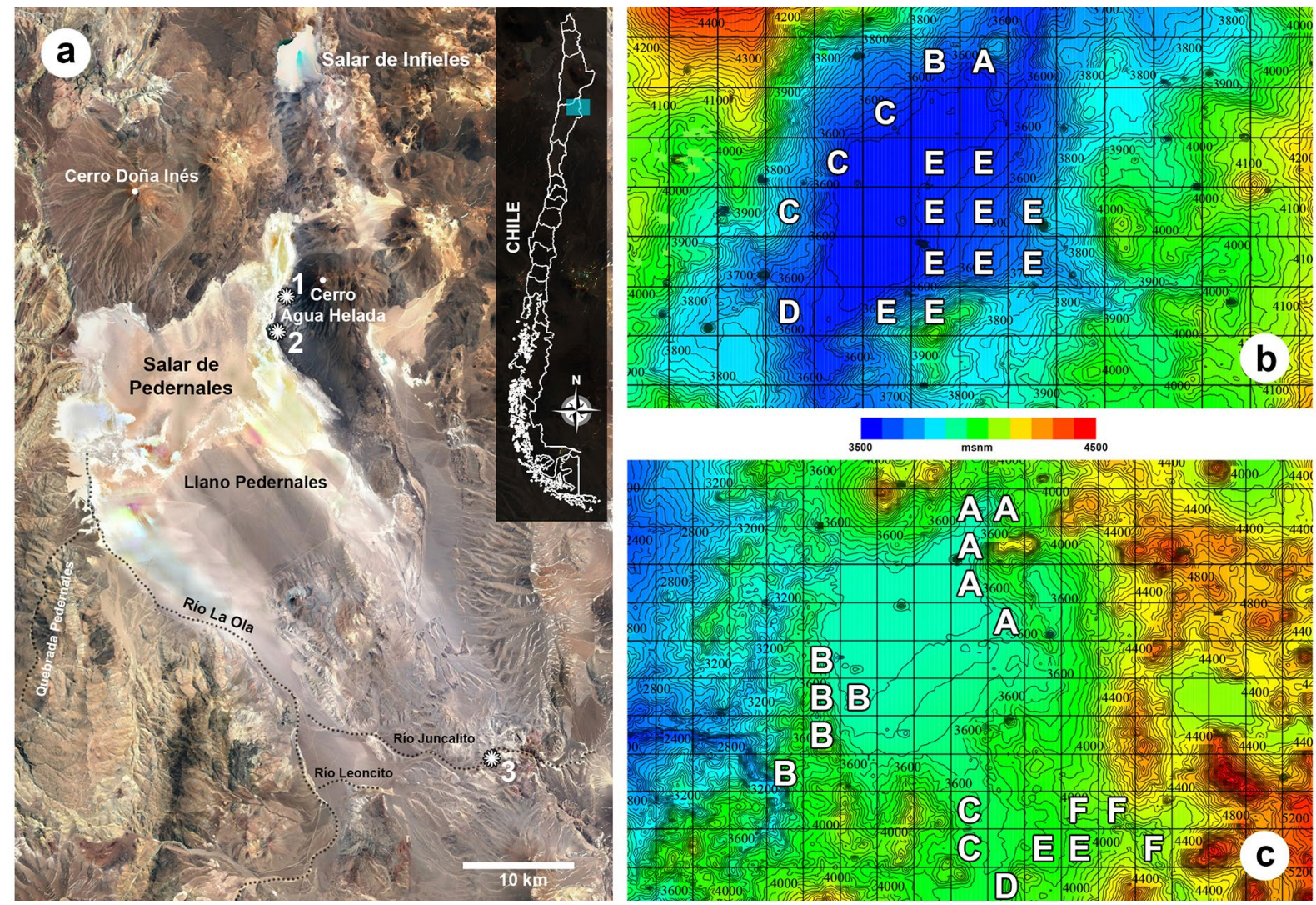

Figura 1. a. Ubicación de los salares de Infieles y Pedernales. Los números indican los sectores de muestreo geoarqueológico de fechados ${ }^{14} \mathrm{C}$; b. Cuadrantes prospectados en Infieles (tamaño de cuadrantes: $1 \mathrm{~km}^{2}$ ); y c. Cuadrantes prospectados en Pedernales (tamaño de cuadrantes: $5 \mathrm{~km}^{2}$ ). 
terrazas y abanicos aluviales del río La Ola; Sector D, cuenca de la quebrada Vega Larga; Sector E, terrazas y vegas del río Leoncito; y Sector $\mathbf{F}$, terrazas del río Juncalito hasta los 4100 msnm (Figura 1c). La distribución espacial de los registros de cronologías prehispánica e indeterminada fueron graficadas mediante la densidad de Kernel, la cual calcula la concentración de hallazgos. El valor es más alto en la ubicación de estos y disminuye a medida que aumenta la distancia desde los hallazgos (Figura 2). Para definir las densidades, se incluyeron también los datos obtenidos en campañas de prospección realizadas por Olivares et al. (2013) para el caso de Pedernales, y que en total suman 20 registros, así como el antecedente de estructuras incaicas en la cima del cerro Doña Inés (5092 msnm).

\section{RESULTADOS}

\section{Aspectos generales del área de estudio}

En general, los ambientes de altura limitan considerablemente la interpretación de contextos arqueológicos debido a una serie de condiciones ambientales y tafonómicas que intervienen en su formación. Algunos factores relevantes son la oscilación térmica, la cobertura de nieve, la radiación solar, ciclos de congelamiento y descongelamiento de los suelos y la erosión eólica. En los salares estudiados, tanto la visibilidad como la accesibilidad del área de estudio son afectadas por los fenómenos aluviales, que se concentran sobre todo en las quebradas que desembocan en cada uno de aquellos. No obstante, las campañas de prospección fueron realizadas pre y poseventos aluviales recientes, y se observaron escasas transformaciones durante cada una de ellas.

La cobertura vegetacional es escasa y no tuvo incidencia en la visibilidad de las evidencias superficiales, la cual fue favorecida por los roedores fosoriales, escorrentía y erosión eólica. La obstrusividad en el caso de los contextos tempranos fue baja debido a que sus registros se componen principalmente de evidencias líticas en materias primas locales, las que en superficie se mezclan con clastos sin alteraciones. Lo anterior no es aplicable para la totalidad de los sitios con dispersiones líticas debido a la alta frecuencia de contextos con materias primas silíceas fácilmente distinguibles en el paisaje por su color, lo mismo que una serie de estructuras asociadas a la conformación de un paisaje para la caza y de otras asociadas al pastoreo.

\section{Salar de Infieles}

En Infieles se contabilizaron un total de 41 registros arqueológicos, entre sitios y hallazgos aislados. De este conjunto, 17 corresponden a evidencias

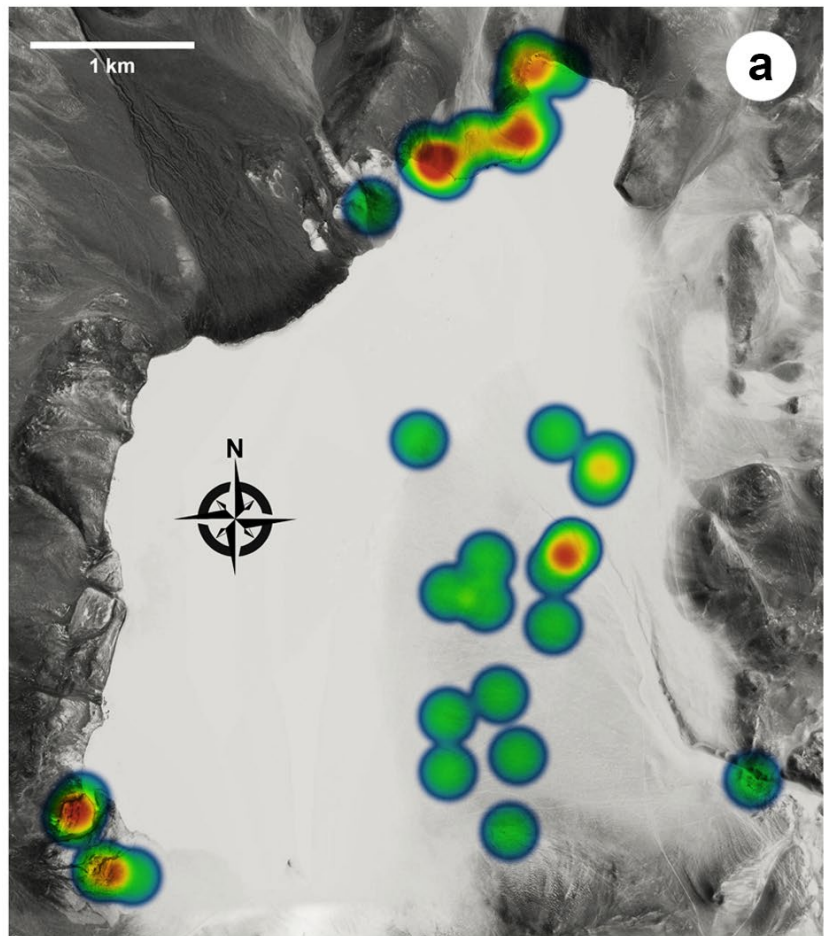

Densidad muy baja

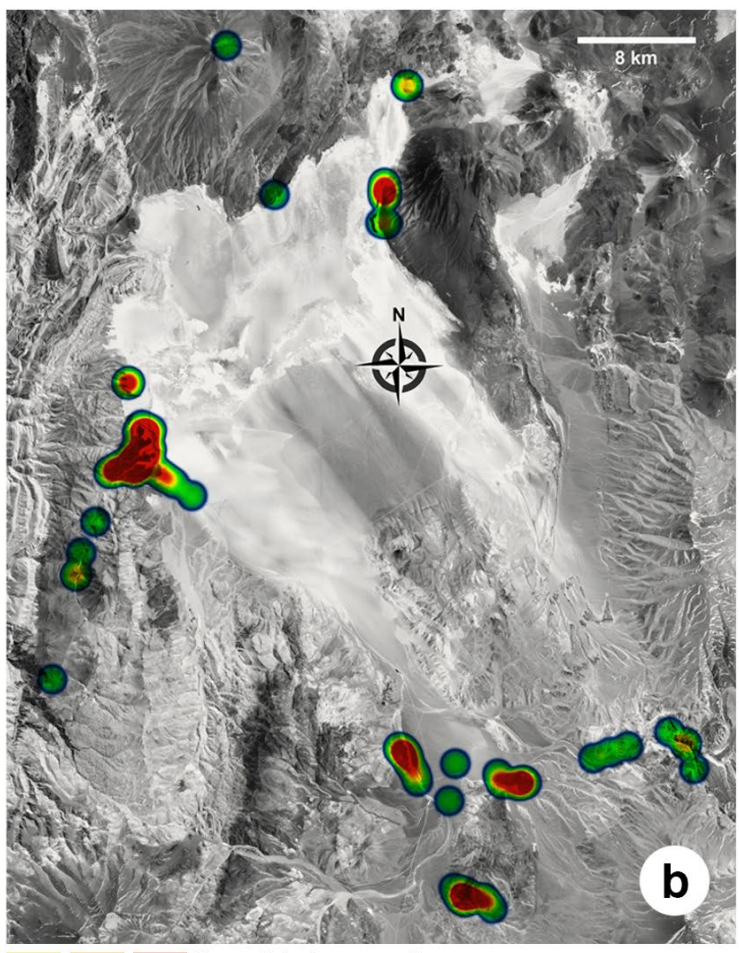

Densidad muy alta

Figura 2. Densidades de Kernel de la distribución de los sitios arqueológicos de: a. Infieles, y b. Pedernales. 
prehispánicas (tres de los cuales son hallazgos aislados), un sitio con rastros tanto históricos como prehispánicos, cuatro sitios históricos y 19 evidencias de cronología indeterminada (Tabla 1). La mayor concentración de rastros prehispánicos se observó en el Sector C; vestigios que corresponden a eventos de talla lítica discretos, emplazados en la playa del salar y compuestos principalmente por desechos primarios y núcleos. En el Sector A destaca el sitio Infieles-19, conformado por una serie de estructuras circulares aglomeradas con una alta densidad de desechos líticos en materias primas silíceas.

Las evidencias prehispánicas más elocuentes de Infieles se encuentran en el afloramiento de ignimbrita (Figura 3a), en cuyos paredones se disponen pinturas rupestres reportadas previamente por Latcham (1938) y Cervellino (1982). En el denominado sitio Infieles-1, fueron registradas una serie de pinturas rojas con estilos adscribibles al periodo Medio afines al estilo Ánimas-La Puerta (1200-800 años cal AP), junto con elementos muy difundidos durante el período Intermedio Tardío (1000-550 años cal AP) (C. Carracedo, comunicación personal, diciembre 2019), y grabados históricos relacionados con procesos de evangelización.

Asociados a estas manifestaciones rupestres, y a los pies de la ignimbrita, se identificó una serie de evidencias arqueológicas tales como fragmentos de cerámica monocroma, desechos líticos, además de restos óseos de vicuñas. Por último, cabe destacar una serie de estructuras

\begin{tabular}{|c|c|c|c|c|c|}
\hline \multicolumn{6}{|c|}{ Salar de Infieles } \\
\hline Sectores & Prehispánico & Histórico-Prehispánico & Histórico & Indeterminado & Total \\
\hline Sector A & 1 & - & - & - & 1 \\
\hline Sector B & 1 & 1 & - & - & 2 \\
\hline Sector C & 8 & - & 1 & 3 & 12 \\
\hline Sector D & 1 & - & 2 & 5 & 8 \\
\hline Sector E & 6 & - & 1 & 11 & 18 \\
\hline Total & 17 & 1 & 4 & 19 & 41 \\
\hline \multicolumn{4}{|c|}{ Altitud promedio } & \multicolumn{2}{|l|}{$3549 \mathrm{msnm}$} \\
\hline \multicolumn{6}{|c|}{ Salar de Pedernales } \\
\hline Sectores & Prehispánico & Histórico-Prehispánico & Histórico & Indeterminado & Total \\
\hline Sector A & 7 & - & 1 & 5 & 13 \\
\hline Sector B & 34 & 9 & 10 & 7 & 60 \\
\hline Sector C & 9 & 1 & 2 & - & 12 \\
\hline Sector D & 7 & 1 & - & 3 & 11 \\
\hline Sector E & 8 & 2 & - & 1 & 11 \\
\hline Sector $\mathrm{F}$ & 2 & 1 & 2 & 5 & 10 \\
\hline Total & 67 & 14 & 15 & 21 & 117 \\
\hline \multicolumn{4}{|c|}{ Altitud promedio } & \multicolumn{2}{|l|}{$3523 \mathrm{msnm}$} \\
\hline
\end{tabular}

Tabla 1. Número de evidencias arqueológicas de los salares de Infieles y Pedernales.
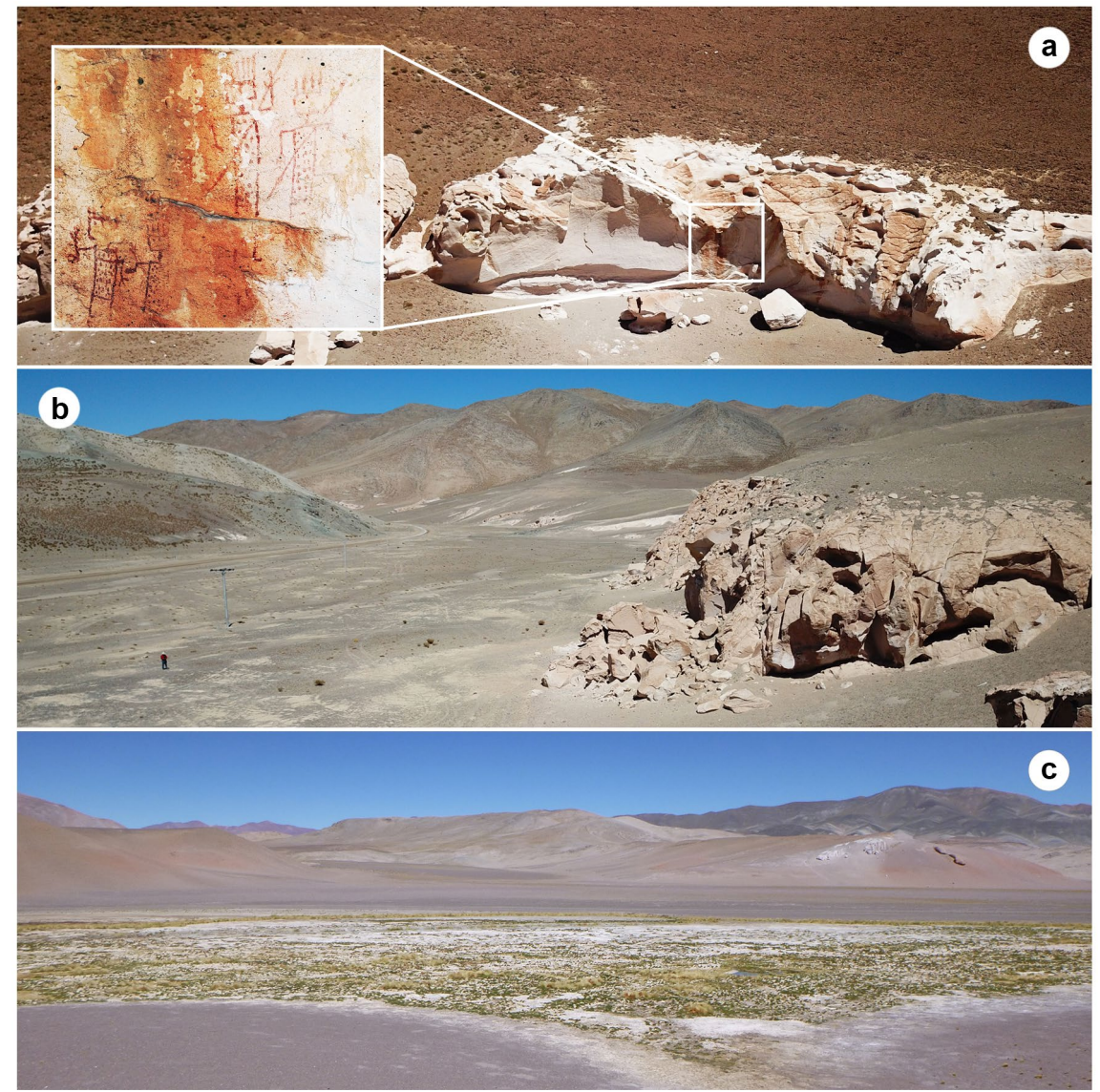

Figura 3. a. Vista del sitio Infieles-1 con detalle de pictografía en uno de sus bloques de ignimbrita; b. Vista del sitio Pedernales-38; c. Vista hacia el este desde el sitio Pedernales-5. Fotografías a y b tomadas por Víctor Méndez. 
de piedra circulares de superficie no mayor a $2 \mathrm{~m}^{2}$ y cuyas alturas no alcanzan un metro. Dichas estructuras de cronología indeterminada se distribuyen en todos los sectores prospectados y se asocian a actividades como la caza, el pastoreo y pernocte.

\section{Salar de Pedernales}

Pese a prospectar una superficie similar en ambas cuencas, el registro arqueológico de Pedernales triplica al de Infieles (Tabla 1). Esto se debe a la mayor diversidad de ambientes en Pedernales, a la alta frecuencia de vertientes aptas para el consumo, cursos fluviales que van en direcciones norte-sur y este-oeste, distintas zonas con fuentes de basaltos aptos para la talla, una mayor diversidad de recursos bióticos y rutas de tránsito hacia pasos cordilleranos.

El Sector A posee características geomorfológicas particulares, puesto que hay una serie de terrazas atribuibles a la expansión o contracción de un ambiente lagunar. La importancia de entender esta dinámica de avance y retroceso del salar radica en ubicar paisajes como paleohumedales actualmente desaparecidos y dirigir parte de las prospecciones al área circundante. Las muestras de sedimentos orgánicos fueron datadas mediante ${ }^{14} \mathrm{C}$ entre 11.340-11.143 años cal AP sobre una terraza ubicada a 3420 msnm (Muestreo 1), y de 24.261 a 23.884 años cal AP en una terraza ubicada a 3350 msnm, a 5 m sobre el nivel actual del salar (Muestreo 2, Figura 4 y Tabla 2). Ahora bien, las prospecciones a lo largo de estas terrazas se vieron dificultadas por la baja visibilidad superficial fomentada por la alta carga de sedimentos transportados por el viento y el aporte sedimentario derivados de rocas volcánicas, lo que dejó visibles únicamente estructuras sin materiales en superficie, salvo una serie de recintos circulares aglomerados emplazados en la base del salar con desechos líticos de sílice en su interior y exterior. La excepción, para el caso de las terrazas más altas, fue el registro de una lasca de basalto altamente patinada con retoque marginal (sitio Pedernales-66), y que guarda preliminarmente una relación tecnológica y tipológica con artefactos del sitio Punta Negra-1, emplazado al sur del Salar de Atacama y cuyas fechas bordean los 12.600 a 10.200 años cal AP (Grosjean et al., 2005).

De los distintos sectores prospectados, el Sector B (quebrada de Pedernales) es el que concentra la mayor cantidad de evidencias arqueológicas prehispánicas. En la actualidad, esta quebrada prácticamente no posee un curso de agua estable debido a las recientes intervenciones mineras. La quebrada se divide en dos áreas: la primera se emplaza hacia el oeste y corresponde a laderas, terrazas fluviales y abanicos aluviales; y la segunda, a geoformas similares junto con el afloramiento de ignimbrita y la desembocadura de la quebrada. Para la primera, los sitios prehispánicos -principalmente dispersiones de desechos líticos y un sitio con estructuras circulares (Pedernales-25) - se emplazan en su mayoría en abanicos aluviales de baja pendiente asociados
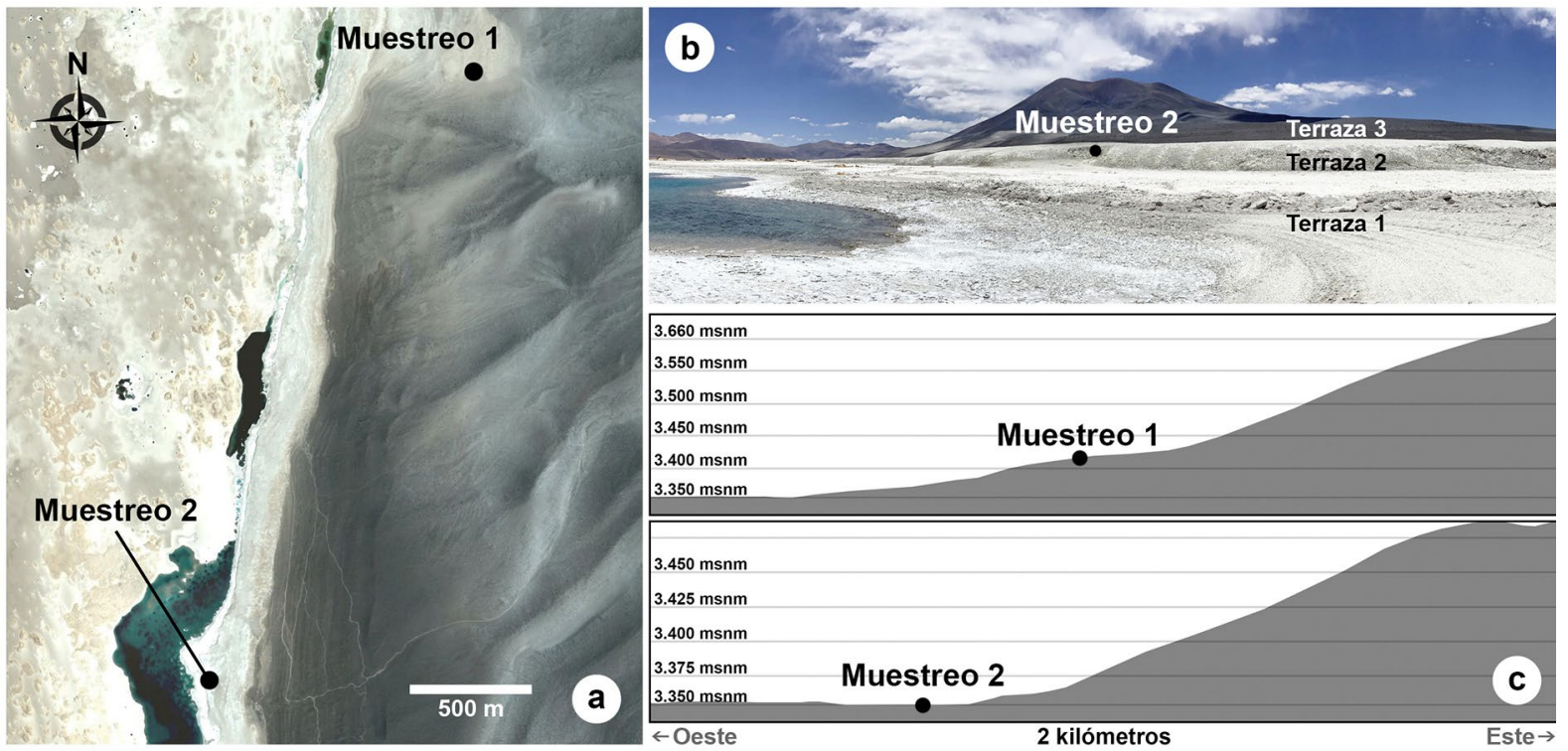

Figura 4. a. Imagen satelital Landsat (Google Earth) con la ubicación de las áreas de muestreo geoarqueológico de sedimentos obtenidos en el Sector A de Pedernales; b. Vista del área muestreada donde se indican las terrazas identificadas en el margen oriental del salar (fotografía tomada por Valentina Flores-Aqueveque); y c. Perfiles de elevación donde se indica la altura de la ubicación de las muestras. 


\begin{tabular}{|c|c|c|c|c|}
\hline \multirow{2}{*}{$\begin{array}{c}\text { Área de } \\
\text { muestreo } \\
\text { geoarqueológico }\end{array}$} & \multirow{2}{*}{$\begin{array}{c}\text { Código } \\
\text { laboratorio }\end{array}$} & Tipo de muestra & $\begin{array}{c}\text { Edad }{ }^{14} \mathbf{C} \text { y } \\
\text { error }\end{array}$ & $\begin{array}{c}\text { Edad }{ }^{14} \mathbf{C} \\
\text { calibrada }\end{array}$ \\
\cline { 4 - 5 } & D-AMS 037296 & Sedimento orgánico & $11.285 \pm 46$ & $11.340-11.143$ \\
\hline 1 & D-AMS 037300 & $\begin{array}{c}\text { Evaporitas con material } \\
\text { orgánico }\end{array}$ & $21.751 \pm 62$ & $24.261-23.884$ \\
\hline 2 & D-AMS 039002 & $\begin{array}{c}\text { Arena con material orgá- } \\
\text { nico (Profundidad 30 cm) }\end{array}$ & $5689 \pm 46$ & $6560-6305$ \\
\hline 3 & D-AMS 039005 & $\begin{array}{c}\text { Arena con material orgá- } \\
\text { nico (Profundidad 30 cm) }\end{array}$ & $4864 \pm 32$ & $5650-5472$ \\
\hline 3 & D-AMS 039004 & $\begin{array}{l}\text { Arena con material orgá- } \\
\text { nico (Profundidad 20 cm) }\end{array}$ & $4699 \pm 42$ & $5573-5305$ \\
\hline 3 & D-AMS 039003 & $\begin{array}{l}\text { Arena con material orgá- } \\
\text { nico (Profundidad 20 cm) }\end{array}$ & $4533 \pm 33$ & $5307-4980$ \\
\hline 3 & D-AMS 039001 & $\begin{array}{c}\text { Arena con material orgá- } \\
\text { nico (Profundidad 30 cm) }\end{array}$ & $3577 \pm 30$ & $3960-3699$ \\
\hline 3 & D-AMS 039006 & $\begin{array}{c}\text { Sedimento fino con ma- } \\
\text { terial orgánico (Profundi- } \\
\text { dad 40 cm) }\end{array}$ & $3072 \pm 37$ & $3361-3077$ \\
\hline
\end{tabular}

Tabla 2. Fechados radiocarbónicos de muestras de sedimentos localizados en el Sector A del Salar de Pedernales. Fechas calibradas mediante OxCal 4.3.2 (Bronk Ramsey, 2017), usando la curva SHCal20 (Hogg et al., 2020). Se debe considerar que las evaporitas potencialmente están afectadas al efecto reservorio.

que Pedernales fue un área que congregó tradiciones culturales afines al núcleo más septentrional.

La segunda área del Sector B corresponde al afloramiento de ignimbrita cuyos paredones ofrecen buenos refugios para el asentamiento humano (Figura 3b), aunque también es un lugar donde se da activamente la "llu-

a vegas activas. Esta tendencia puede deberse a la filtración de agua a partir de corrientes dispersivas radiales que descienden por los abanicos y que alimentan a las vegas aledañas, lo que genera ambientes altamente productivos. La calidad de las materias primas identificadas en estos sitios es óptima, con rocas silíceas cuyas fuentes no se encuentran en las áreas prospectadas. El sitio Pedernales-25 posee características arquitectónicas afines a grupos arcaicos tardíos con recintos circulares aglomerados de diámetro no superior a $2 \mathrm{~m}$ y con una alta densidad de desechos líticos de sílice, entre los que se incluye la base de una punta de proyectil apedunculada. Asimismo, hallazgos aislados de cerámica tipo Los Morros en la misma quebrada, profusamente registrada en el Loa MedioSuperior y en los oasis del Salar de Atacama (Núñez et al., 2017), apuntan a
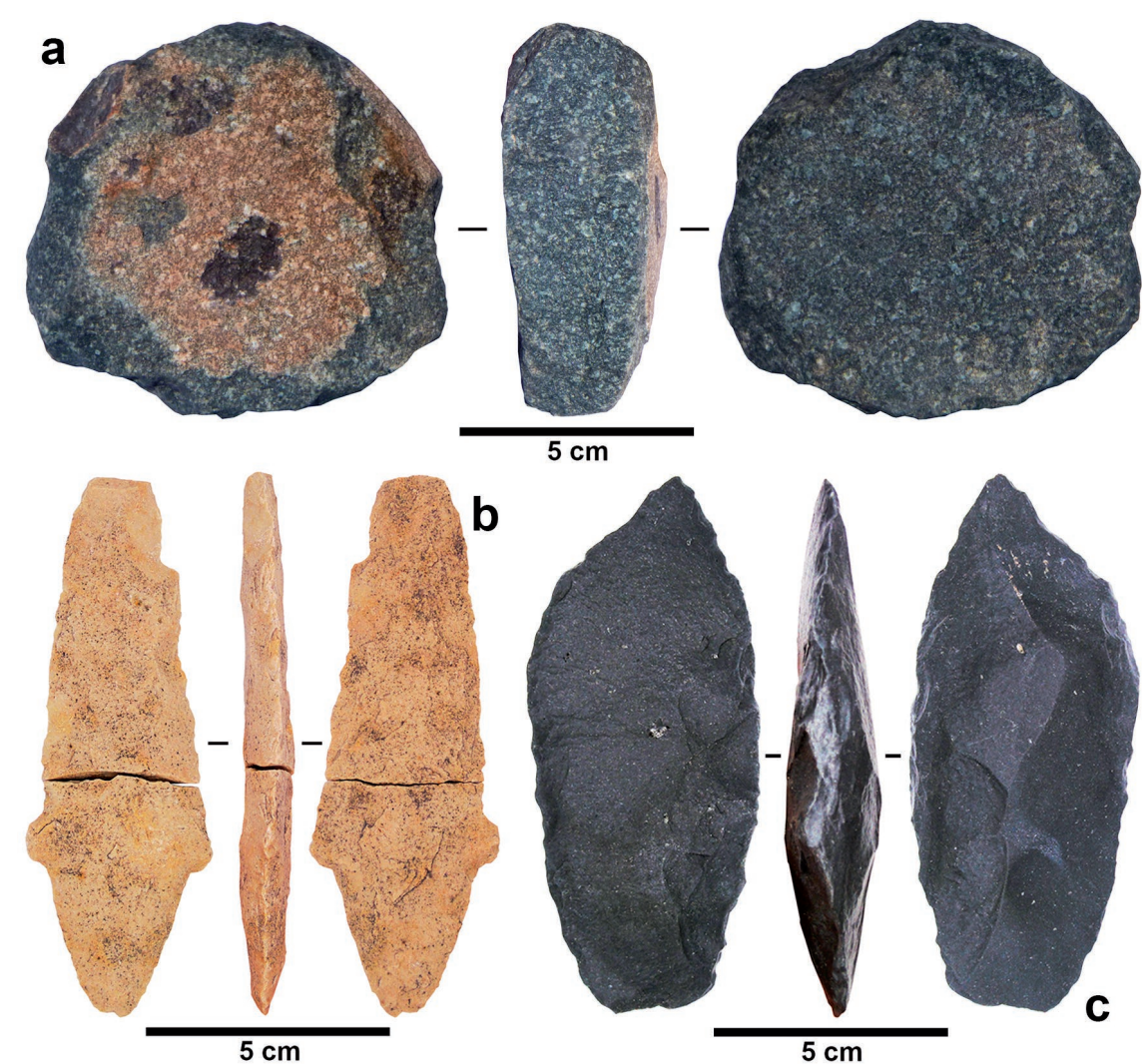

Figura 5: a. Preforma de posible lito geométrico asociado del sitio Pedernales-39; b. Punta pedunculada registrada en superficie en el sitio Pedernales-38; c. Lasca con retoque bimarginal del sitio Pedernales-5. 
se identificaron hallazgos aislados de preformas de litos geométricos afines al Complejo Cultural Huentelauquén (Figura 5a), ampliamente estudiado en el Norte Semiárido y Árido de la costa chilena, con fechas que abarcan desde los 12.680 hasta los 7750 años cal AP (Llagostera et al., 2000; Jackson et al., 2011) y con escasa presencia en valles y zonas cordilleranas (Escudero et al. 2016). Por otra parte, en el sitio Pedernales-38, a 20 m de la preforma de lito, se hallaron diversas puntas de proyectil pedunculadas, similares a las registradas para Huentelauquén, Patapatane (9500-8000 años cal AP; Osorio et al., 2011) y Las Cuevas, ambas en la puna seca (10.412-11.240 años cal AP; Osorio, 2013) (Figura 5b).

Del sitio Pedernales-38 se reconocieron, en un área de $1300 \mathrm{~m}^{2}$, cerca de 50 fragmentos de puntas de proyectil peducunladas, entre los que se incluyen artefactos terminados y preformas en distintas fases de manufactura, además de raspadores discoidales y desechos de talla. Del conjunto de fragmentos de puntas terminadas, casi la totalidad corresponden a fragmentos de pedúnculos con fracturas transversales y oblicuas sobre las aletas. En la confección de estas piezas domina un vidrio volcánico de origen desconocido, junto con andesitas de grano grueso para la talla y disponibles en la quebrada a partir de rodados. Tales características y la alta densidad de artefactos y desechos líticos proponen a Pedernales-38 -sitio que está en proceso de estudio- como un campamento temprano donde se realizaron tareas relacionadas con el descarte y reemplazo de puntas datado en 10.151-9695 años cal AP (Tabla 3).

A lo largo de este sector y en toda la cuenca de Pedernales, diversas estructuras de piedra semicirculares (posibles "mesadas" sensu Moreno y Quesada, 2012) con desechos de talla de sílices y que apenas sobresalen del nivel de suelo, sugieren una conformación de paisajes de caza articulados con los campamentos principales.

En el Sector C, la alta densidad de sitios se explica por la formación de vegas asociadas al río La Ola. La mayor parte de estas evidencias corresponden a ocupaciones alfareras, con una alta densidad de material, que incluye ejemplares de Felicioliva peruviana. Estos sitios se emplazan en las terrazas aledañas a las cuencas con aportes sedimentarios fluviales y eólicos, por lo que, para evaluar ocupaciones tempranas, se prospectaron abanicos aluviales no obliterados por depósitos holocénicos recientes (Figura 3c). Esto permitió identificar un extenso campamento (Pedernales-5) con una serie de acumulaciones de rocas, núcleos y derivados en fases iniciales de extracción in situ, que están distanciados entre sí unos 15 a 20 m. El único artefacto registrado corresponde a una gran lasca cortical de basalto negro altamente patinada, extraída mediante percusión directa con un filo doble convergente en punta (Figura 5c). El ángulo cercano a

\begin{tabular}{|c|c|c|c|c|}
\hline $\begin{array}{l}\text { Sitio/ } \\
\text { Hallazgo } \\
\text { aislado }\end{array}$ & Sector & Altitud & Cronología & Artefactos diagnósticos \\
\hline Pedernales-3 & Sector $\mathrm{E}$ & 3731 & $\begin{array}{l}\text { Arcaico temprano- } \\
\text { Alfarero-Histórico }\end{array}$ & Punta de proyectil triangular tipo Tuina. \\
\hline Pedernales-4 & Sector E & 3668 & Arcaico temprano & Lámina de basalto con retoque unilateral-bimarginal. \\
\hline Pedernales-5 & Sector B & 3621 & Arcaico temprano & $\begin{array}{l}\text { Lasca de basalto con retoque unilateral-bimarginal } \\
\text { asociada a extenso campamento acerámico. }\end{array}$ \\
\hline Pedernales-22 & Sector B & 3377 & ¿Arcaico temprano? & $\begin{array}{l}\text { Posible preforma de lito geométrico afín al Comple- } \\
\text { jo Cultural Huentalauquén, asociado a restos líticos } \\
\text { (lascas). }\end{array}$ \\
\hline Pedernales-37 & Sector B & 3368 & Arcaico temprano & $\begin{array}{l}\text { Punta pedunculada en basalto aislada en bloque de } \\
\text { ignimbrita. }\end{array}$ \\
\hline Pedernales-38 & Sector B & 3367 & $\begin{array}{l}\text { Arcaico temprano } \\
10.151-9695 \text { años } \\
\text { cal AP }\end{array}$ & $\begin{array}{l}\text { Múltiples pedúnculos de puntas de proyectil, bifaces } \\
\text { y raspadores discoidales. }\end{array}$ \\
\hline Pedernales-39 & Sector B & 3368 & $\begin{array}{l}\text { Arcaico temprano- } \\
\text { Histórico subactual }\end{array}$ & $\begin{array}{l}\text { Posible preforma de lito geométrico afín al Complejo } \\
\text { Cultural Huentalauquén, asociado a restos líticos. }\end{array}$ \\
\hline Pedernales-66 & Sector A & 3386 & ¿Arcaico temprano? & Lasca de basalto con retoque unilateral-bimarginal. \\
\hline Pedernales-84 & Sector C & 3664 & ¿Arcaico temprano? & $\begin{array}{l}\text { Posible preforma de lito geométrico afín al Complejo } \\
\text { Cultural Huentalauquén. }\end{array}$ \\
\hline
\end{tabular}

Tabla 3. Sitios con afiliación temprana (Pleistoceno tardío-Holoceno temprano) registrados en el Salar de Pedernales. 
$50^{\circ}$ del bisel, la sección planoconvexa y la morfología del filo sugieren que este artefacto fue utilizado como cuchillo-raedera. Lo interesante de esta pieza es que presenta similitudes con el uso de grandes lascas corticales en otros contextos arqueológicos tempranos como los sitios de Punta Negra-1, TaguaTagua-2 y Tuina-5, de donde se recobraron raederas similares confeccionadas sobre basalto (Núñez et al., 1994, 2002; Loyola et al., 2017).

En el Sector $\mathbf{E}$, las evidencias se relacionan también con la explotación de los recursos disponibles de la vega formada por las aguas de los ríos Leocinto y Juncalito, incluyendo sitios del periodo Tardío o influencia incaica, periodo Intermedio Tardío (Diaguita) y ocupaciones arcaicas. En un abanico aluvial, cuya descarga desciende hacia la vega del Leoncito, se identificaron puntas de proyectil apedunculadas del tipo Tuina y Tambillo, hallazgo aledaño a un campamento con ocupaciones tanto alfareras como arcaicas (Pedernales-3). La fase Tuina posee fechas que van de los 11.000 a los 9500 años cal AP y representa a cazadores andinos con patrones de alta movilidad, con sitios emplazados entre ca. 3000 y 4200 msnm (Núñez, Grosjean y Cartagena, 2005). La denominada tradición TuinaInca Cueva para los salares más australes es, hasta la fecha, desconocida, y se han identificado posibles evidencias superficiales para la cuenca alta del río Jorquera $\left(27^{\circ} \mathrm{S}, \mathrm{R}\right.$. Loyola, comunicación personal, diciembre 2019), así como en la vertiente atlántica en el sector del valle de Las Taguas $\left(29^{\circ} \mathrm{S}\right)$ (Cortegoso, 2014). Pese a que esta morfología de puntas se da a lo largo de toda la secuencia holocénica, ciertos rasgos tecnológicos en el ejemplar de Pedernales-3 apuntan a un sustrato tecnológico común para grupos tempranos, como la presencia de "esquillamiento" sobre el limbo realizado por percusión apoyada.

Por último, en el Sector $\mathbf{F}$, siguiendo el curso medio-alto del río Juncalito, el encajonamiento de la quebrada no favoreció el registro arqueológico, salvo por hallazgos históricos y estructuras de escasas dimensiones de cronologías indeterminadas. En este sector, el muestreo geoarqueológico de depósitos orgánicos en el curso medio del río Juncalito (Muestreo 3, $3880 \mathrm{msnm}$ ) indica la formación de humedales hacia el Holoceno medio y tardío con fechas ${ }^{14} \mathrm{C}$ que van desde los 6560-6305 hasta los 3361-3077 años cal AP (Tabla 2). Estas dataciones son coherentes con la ausencia de contextos arqueológicos del Pleistoceno tardío-Holoceno temprano hacia los cursos medio y alto de esta cuenca.

\section{DISCUSIÓN Y CONCLUSIONES}

En áreas como los salares de Pedernales e Infieles, dos grandes escenarios para grupos cazadores-recolectores son plausibles en cuanto al uso del espacio. Por un lado, bases residenciales con ocupaciones relativamente prolongadas en las cuales se llevaron a cabo diversas actividades; mientras que un segundo escenario apunta al uso de esta área por tiempos breves para actividades como la caza, recolección (incluyendo huevos), la obtención de pieles de alta calidad, como las de vicuña, y de materias primas; o bien como áreas de pastoreo trashumante para momentos tardíos. En el caso de la fauna, ambientes emplazados sobre los $3000 \mathrm{msnm}$ son menos productivos $\mathrm{y}$, en teoría, menos predecibles. No obstante, especies como la vicuña -abundante en la actualidad en Pedernales e Infieles-, por su requerimiento diario de agua, son fácilmente localizables en quebradas y vegas. Por ende, en una fase de exploración inicial en ambientes como Infieles y Pedernales, especies como la mencionada disminuyen el riesgo de la caza en espacios en franco proceso de conocimiento y es esperable su registro a lo largo de toda la secuencia.

Las evidencias líticas -debido a su mayor visibilidad y abundancia en ambos salares en esta fase de prospección- permiten discutir algunas de nuestras expectativas sobre el proceso de poblamiento en escalas espaciales y temporales más amplias. Para una etapa de exploración inicial, en la cual se esperan ocupaciones discontinuas, con una movilidad centrada en rutas naturales y un desconocimiento de la totalidad de los recursos bióticos y abióticos disponibles en los ambientes explorados (Borrero, 1989-1990), se han formulado expectativas reflejadas en el uso de materias primas, frecuencia de artefactos, el transporte de estos, etc. Así, Borrero y Franco (1997) plantearon, entre diversas expectativas, la abundancia de artefactos expeditivos; la baja frecuencia de artefactos curados; el reensamblaje de desechos producidos en fases terminales de elaboración de artefactos transportados; una alta frecuencia de uso de materias primas locales y un bajo uso de materias primas foráneas; el transporte de preformas o artefactos ya terminados; y el uso de rocas de mediana a baja calidad, en el caso de áreas con ausencia de materias primas de calidad óptima para la taIla. Para las evidencias tempranas de Pedernales o tentativamente asignadas por sus tipologías líticas a los primeros momentos del Holoceno, dominan 
materias primas locales muestreadas e inmediatamente disponibles (andesitas y basaltos) y las que se diferencian claramente de contextos tardíos con materias primas óptimas para la talla, como sílices y obsidianas, cuyas fuentes, a la fecha, desconocemos. Las andesitas y basaltos se encuentran disponibles en la totalidad de las quebradas, abanicos aluviales y cursos fluviales estudiados y, por tanto, limitan el transporte de preformas a sitios articulados, salvo el caso de vidrios volcánicos registrados en Pedernales-38, no disponibles en bloques de tamaños adecuados para la elaboración de puntas como las identificadas en este sitio. Siguiendo a Franco (2002), en una fase de exploración se prevé que los núcleos de materias primas locales posean pocas extracciones, como es el caso de los soportes líticos de Pedernales-5, sitio en donde también hay un abundante registro de lascas primarias y cuyos tamaños superan a las observadas en contextos tardíos. La contrastación de estas expectativas requerirá de un mayor análisis de los contextos registrados. Más importante aún, será necesario relacionar las evidencias con otras tradiciones culturales tempranas contemporáneas: Huentelauquén, Patapatane, Tuina Inca-Cueva y Punta Negra.

Como planteamos al inicio de este trabajo, una de nuestras hipótesis es que las tierras altas meridionales fueron espacios internodales de tránsito entre las vertientes del Pacífico y Atlántico, a lo que agregamos el tránsito norte-sur de cazadoresrecolectores con tradiciones culturales distintas, pero cuyas cronologías presentan rangos solapados. Esto nos lleva a formular nuevas preguntas sobre la ocupación sincrónica hacia el Holoceno temprano de los espacios en los salares meridionales. Un escenario plausible es que estas ocupaciones correspondan a cazadores-recolectores Huentelauquén (a decir por la posible preforma de lito geométrico y morfología de puntas pedunculadas), lo cual trae consigo el interrogante de cómo y por qué bandas de cazadores-recolectores con un conocimiento profundo de ambientes costeros comenzaron a explorar la precordillera y las tierras altas. Otro escenario, que no excluye al anterior, es que las evidencias registradas dieran cuenta de tradiciones tempranas de tierras altas también portadoras de puntas pedunculadas, como Patapatane y Las Cuevas. Finalmente, también podría tratarse de manifestaciones tecnológicas y culturales locales, aspecto que deberá ser precisado a futuro tras estudios tecnológicos sistemáticos.
A modo de conclusión, la búsqueda de sitios tempranos en las cuencas de los salares de Infieles y Pedernales fue efectiva para esta segunda cuenca. Estos registros se ajustan parcialmente a expectativas de grupos que exploraron inicialmente estos ambientes, aunque dichas expectativas requieren de una necesaria calibración con un área que arqueológicamente es Terra Incognita. Lo anterior apunta a valorizar más en futuras prospecciones sectores como desembocaduras de quebradas, terrazas fluviales y, sobre todo, abanicos aluviales, incluso por sobre las playas de salares estudiadas profusamente en los salares septentrionales (Loyola, Núñez y Cartajena, 2019). Por último, futuros trabajos también deberán enfocarse a problemas similares a los del Norte Árido, ya que estudios paleoclimáticos en la laguna Negro Francisco indican eventos de estrés ambiental y condiciones húmedas sincrónicas a las descritas para el núcleo nortino (Grosjean et al., 1997), por lo que resulta de gran interés comparar las trayectorias culturales a lo largo de la secuencia holocénica. Esta primera fase de prospección será seguida por otra de sondeos arqueológicos que enfaticen los hallazgos realizados y sectores como aleros, cuyas cualidades como reservorios estratigráficos permitirán comprender mejor la cronología y comportamiento de las diversas ocupaciones arqueológicas de ambas cuencas.

\section{Agradecimientos}

El presente trabajo fue financiado por la Agencia Nacional de Investigación y Desarrollo de Chile a través del Proyecto Fondecyt 1190197: "Ocupaciones humanas y paisajes de altura. Marco paleoambiental y geoarqueológico durante el poblamiento humano inicial en la puna meridional del desierto de Atacama (26-28º S, 3.200-4.500 msnm)". Nuestro agradecimiento a los(as) colegas arqueólogos(as), antropólogos(as) y geólogos(as) que participaron en los trabajos de campo y escritorio: Elvira Latorre Blanco, Álvaro Bravo, Ignacio Álamos, Víctor Méndez, Consuelo Carracedo, Anahí Maturana, Úrsula Osses, Francisco Pérez, Pedro Hueche y Angélica Soto. Agradecemos, además, a la Comunidad colla Diego de Almagro por todo su apoyo durante los trabajos de terreno. Por último, agradecemos a todos(as) los(as) evaluadores(as), cuyos comentarios permitieron mejorar una primera versión de este trabajo. 


\section{REFERENCIAS CITADAS}

Albarracin-Jordan, J. y Capriles, J. (2011). The Paleoamerican occupation of Cueva Bautista: latePleistocene human evidence from the Bolivian highlands. Current Research in the Pleistocene, 28, 95-98.

Aldenderfer, M. (1998). Montane foragers. Asana and the south central andean Archaic. University of lowa Press.

Algoritmos Consultores (2011). Informe prospección arqueológica reapertura operación Agua de la Falda, Proyecto Jerónimo. http://bibliotecadigital. ciren.cl/bitstream/handle/123456789/6844/ MMA-HUM2_0007_v3.pdf?sequence=1 \&isAllowed =y

Binford, L. (2001). Constructing frames of reference: an analytical method for archaeological theory building using ethnographic and environmental data sets. University of California Press.

Borrero, L. A. (1988). Tafonomía Regional. En N. Ratto y A. Haber (Eds.), De procesos, contextos y otros huesos (pp. 9-15). Universidad de Buenos Aires.

Borrero, L. A. (1989-1990). Evolución cultural divergente en la Patagonia austral. Anales del Instituto de la Patagonia, Serie Ciencias Humanas, 19, 133-140.

Borrero, L. A. y Franco, N. (1997). Early patagonian hunter-gatherers: subsistence and technology. Journal of Anthropological Research, 53(2), 219-239.

Bronk Ramsey, Ch. (2017). Methods for summarizing radiocarbon database. Radiocarbon, 59(2), 1809-1833.

Capriles, J. y Albarracin-Jordan, J. (2013). The earliest human occupations in Bolivia: a review of the archaeological evidence. Quaternary International, 301, 46-59.

Capriles, J. Albarracin-Jordan, J., Lombardo, U., Osorio, D., Maley, B., Goldstein, S., Herrera, K., Glascock, M., Domic, A., Veit, H. y Santoro, C. (2016). High-altitude adaptation and late Pleistocene foraging in the Bolivian Andes. Journal of Archaeological Science: Reports, 6, 463-474.

Castelleti, J. (2011). Prospección arqueológica y paleontológica. Proyecto "Flotación de Escorias Convertidor Teniente Fundición Potrerillos", Codelco División Salvador Rev. 2. Fundación Chile. http://bibliotecadigital. ciren.cl/bitstream/handle/123456789/7132/ MMA-HUM2_0075_v2.pdf?sequence=1

Cervellino, M. (1982). Salares del norte, Salar de Infieles. Revista Creces: http://www.creces.cl/Contenido?art=784

Cortegoso, V. (2014). Valle de las Taguas, ARQ-18: estratigrafía, secuencia temporal y ocupaciones humanas. En V. Cortegoso, V. Durán y A. Gasco (Eds.),
Arqueología de Ambientes de Altura de Mendoza y San Juan (Argentina) (pp. 209-242). Mendoza, Argentina: EDIUNC.

Escudero, A., Dávila, C., Villela, F., Troncoso, A., Méndez, C. y López, P. (2016). Early Holocene inland occupation in the Semiarid North of Chile. Paleoamerica, 2(1), 74-77.

Franco, N. (2002) ¿Es posible diferenciar los conjuntos líticos atribuidos a la exploración de un espacio de los correspondientes a otras etapas del poblamiento? El caso del extremo sur de Patagonia. Revista Werkén, 3, 119-132.

Gamble, C. (1993). Timewalkers. Cambridge University Press.

Grosjean, M., Valero-Garcés, B., Geyh, M., Messerli, B., Schotterer, U., Schreier, H. y Kelts, K. (1997). Mid and late Holocene limnogeology of Laguna del Negro Francisco, northern Chile, and its paleoclimatic implications. The Holocene, 7(2), 151-159.

Grosjean, M., Núñez, L. y Cartajena, I. (2005) Paleoindian occupation of the Atacama Desert, Northern Chile. Journal of Quaternary Science, 20(7-8), 643-653.

Hogg, A., Heaton, T., Hua, Q., Palmer, J., Turney, C., Southon, J., Bayliss, A., Blackwell, P., Boswijk, G., Bronk Ramsey, C., Pearson, C., Petchery, F., Reimer, P., Reimer, R. y Wacke, L. (2020) SHCal20 southern hemisphere calibration, 0-55,000 years cal BP. Radiocarbon, 62(4), 759-778.

Jackson, D., Maldonado, A., Carré, M. y Seguel, R. (2011). Huentelauquén Cultural Complex: the earliest peopling of the Pacific coast in the South-American southern cone. En D. Vialou (Ed.), Peuplement et Préhistoire en Amériques (pp. 221-231). Éditions du Comité des Travaux Historiques et Scientifiques.

Kelly, R. (1995). The foraging spectrum. Diversity in huntergatherer Lifeways. Smithsonian Institution Press.

Kelly, R. (2003). Colonization of new land by huntergatherers: expectations and implications based on ethnographic data. En M. Rockman y J. Steele (Eds.), Colonization of unfamiliar landscapes: the archaeology of adaptation (pp. 44-59). Routledge.

Kull, C., Grosjean, M. y Veit, H. (2002). Modelling modern and Late Pleistocene glacio-climatological conditions in the North chilean Andes ( $\left.29^{\circ} \mathrm{S}-30^{\circ} \mathrm{S}\right)$. Climate Change, 52, 359-381.

Latcham, R. (1938). Arqueología de la región Atacameña. Universidad de Chile.

Latorre, C., Santoro, C., Ugalde, P., Gayó, E., Osorio, D., Salas-Egaña, C., De Pol-Holz, R., Joly, D. y Rech, J. (2013). Late Pleistocene human occupation of the hyperarid core in the Atacama Desert, northern Chile. Quaternary Science Reviews, 77, 19-30. 
Llagostera A., Weisner, R., Castillo, G., Cervellino, M. y Costa-Junqueira, M. (2000). El Complejo Huentelauquén bajo una perspectiva macroespacial y multidisciplinaria. Contribuciones Arqueológicas Museo Regional de Atacama, 5, 461-480.

Loyola, R., Núñez, L., Aschero, C. y Cartajena, I. (2017) Tecnología lítica del Pleistoceno final y la colonización del Salar de Punta Negra $\left(24,5^{\circ} \mathrm{S}\right)$, Desierto de Atacama. Estudios Atacameños, 55, 5-34.

Loyola, R., Núñez, L. y Cartajena, I. (2019). What's like out there? Landscape learning during the early peopling of the highland of the south-central Atacama desert. Quaternary International, 53(20), 7-24.

Maldonado, A., Betancourt, J., Latorre, C. y Villagrán, C. (2005). Pollen analyses from a 50.000-yr rodent midden series in the southern Atacama Desert (25 30' S). Journal of Quaternary Science, 20(5), 493-507.

Moreno, E. y Quesada, M. (2012). Construcción de paisajes de caza y relaciones sociales de larga duración en Antofalla. En P. Babot, M. Marschoff y F. Pazzarelli (Eds.), Las manos en la masa. Arqueologías y Antropologías de la alimentación en Suramérica (pp. 583-604). Universidad Nacional de Córdoba, Museo de Antropología.

Núñez, L., Varela, J., Casamiquela, R., Schiappacasse, V., Niemeyer, H. y Villagrán, C. (1994). Cuenca de Taguatagua en Chile: el ambiente del Pleistoceno superior y ocupaciones humanas. Revista Chilena de Historia Natural, 67, 503- 519.

Núñez, L., Grosjean, M. y Cartajena, I. (2002). Human occupations and climate change in the Puna de Atacama, Chile. Science, 298, 821-824.

Núñez, L, Grosjean, M. y Cartagena, I. (2005). Ocupaciones humanas y paleoambientes en la Puna de Atacama. Universidad Católica del Norte; Taraxacum.
Núñez, L., Cartajena, I., Carrasco, C., López, P., Rivera, F., de Souza, P., Santander, B. y Loyola, R. (2017). Presencia de un centro ceremonial Formativo en la circumpuna de Atacama. Chungara, 49(1), 3-33.

Olivares, F., Amacay de Atacama Cepeda, Z., Troncoso, I., Troncoso, T. y Fernández, F. (2013). Caracterización arqueológica. Informe de caracterización biológica general y definición de metodologías de monitoreo para seguimiento en el sitio prioritario de la biodiversidad Salar de Pedernales y sus alrededores. http://bdrnap. mma.gob.cl/recursos/SINIA/Biblio\%20SP-64/92.pdf

Osorio, D. (2013). Reevaluación del Arcaico Temprano de la Puna Seca: ( 12.000 años cal. AP- 9.000 años cal. AP). Implicancias para el poblamiento Inicial del altiplano del Norte Grande de Chile (tesis de pregrado). Universidad de Chile, Santiago, Chile.

Osorio, D., Jackson, D., Ugalde, P., Latorre, C., de PolHolz, R. y Santoro, C. (2011). Hakenasa cave and its relevance for the peopling of Andean Altiplano. Antiquity, 85, 1194-1208.

Quade. J., Rech, J., Betancourt, J., Latorre, C., Quade, B., Aasen Rylander, K. y Fisher, T. (2008). Paleowetlands and regional climate change in the central Atacama Desert, northern Chile. Quaternary Research, 69, 343-360.

Rademaker, K., Hodgins, G., Moore, K., Zarrillo, S., Miller, C., Bromley, G., Leach, P., Reid, D., Yépez, W. y Sandweiss, D. (2014). Paleoindian settlement of the high-altitude Peruvian Andes. Science, 346, 466-469.

Yacobaccio, H. (2017). Peopling of the high Andes of northwestern Argentina. Quaternary International, 461, 34-40. 
